\title{
Contributions to the Analysis and the Supervision of a Thermal Power Plant
}

\author{
Lakhoua M.N \\ The National Engineering School of Carthage, \\ University of Carthage, Research Unit: \\ Signals \& Mechatronic Systems, Tunisia \\ Glaa R. \\ The National Engineering School of Carthage, \\ University of Carthage, Research Unit: \\ Signals \& Mechatronic Systems, Tunisia
}

\author{
Ben Hamouda M. \\ The National Engineering School of Carthage, \\ University of Carthage, Research Unit: \\ Signals \& Mechatronic Systems, Tunisia \\ El Amraoui L. \\ The National Engineering School of Carthage, \\ University of Carthage, Research Unit: \\ Signals \& Mechatronic Systems, Tunisia
}

\begin{abstract}
Supervision systems play an important role in industry mainly due to the increasing demand for product quality and high efficiency, and to the growing integration of automatic control systems in technical processes. In fact, the supervision system has a great number of components and interconnections, and it is difficult to describe and understand its behavior. Furthermore, the supervision system in industrial plants, implemented in supervisory control and data acquisition (SCADA) software, must undertake, at least, the following three main tasks: monitoring, control and fault tolerance. So it can be classified as a complex system. The objective of this paper is to show interests of the use of functional analysis techniques such as SADT (Structured Analysis and Design Technique) and SA-RT (Structured Analysis Real Time) for the design of supervisory systems. This is why we present a general model of analysis and supervision of production systems. This model was based on the one hand on the functional analysis (FA) and on the other hand on the SCADA system.
\end{abstract}

Keywords-SCADA systems; SADT method; SA-RT method; thermal power plant

\section{INTRODUCTION}

In our days in different industrial areas it become very important to analysis, control and supervise the production systems. In fact, the most used are supervisory control and data acquisition (SCADA) systems; these are used to control systems in different areas, to collect information and to centralized data acquisition. One of the most important concerns in the SCADA design is to assure whether the system could go with the reliability and performance requirements specification.

The production systems of increasing complexity present difficulties at the level of their functional analyses. Several types of functional and structural analysis methods exist and are used in many industrial domains since about thirty years. In fact, the functional analysis (FA) permits the synthetic description of fashions of working of a system and the knowledge of functions to guarantee [1]. It establishes of systematic and exhaustive way the functional relations inside and outside of the system. Again, the FA consists in searching for and to characterize functions offered by a system to satisfy its user's needs.

The objective of FA methods is to describe and to understand the system. It is gaits that put the accent on what makes the system, to master the complexity and to detect defaulting. So the FA serves to model the system while clearing constraints and while specifying the different fashions of working [1].

Many well-known graphical modeling techniques, for example, the Structured Analysis Real Time (SA-RT) method developed by Hatley and Pirbhai [2], provide mechanisms that allow one to: (1) abstract over details best left to later stages of development, (2) model an application along different views, and (3) modularize problems and solutions. It has also been affirmed that the simple and graphical manner of the modeling constructs facilitates the construction of concise and understandable models [3].

This paper can be loosely divided into five parts: first, we summarize the complexity of supervision systems particularly to design SCADA systems and second briefly describe the characteristics of a SCADA system and the problems related to its design. Subsequently, we present the issues involved in the analysis and supervision of the production systems. In section 3 , after presenting concepts of SADT and SA-RT, a model of analysis and supervision of production systems is presented. Then, the benefits of using SADT and SA-RT in the design steps are developed. Next, the results of the proposal methodology was used on a case study of a SCADA system of a thermal power plant (TPP) particularly in programming and visualizing new chemical analysis parameters of the water steam cycle. In the last section, we present a discussion about the advantages and inconveniences of the methodology used in order to describe and understand the behavior of supervision systems.

\section{DESIGN OF SCADA SYSTEMS}

With the advances of electronic and software technologies, the SCADA systems are generally used in industrial plant automation. It provides an efficient tool to monitor and control 
equipment in industrial processes. In fact, SCADA systems can be found in critical infrastructures such as power plants [4] [5] and power grid systems, water, oil and gas distribution systems, building monitoring, production systems and other products.

In a typical SCADA system, data acquisition and control are performed by remote terminal units (RTUs) and field devices that include functions for communications and signaling. In fact, the control performed by SCADA is much more superficial and generally applied to correct a fault and the SCADA system monitors and provide control actions based on this monitoring [6].

The whole automation process is done using programmable logic controller (PLC) which has number of unique advantages like speed, reliability, less maintenance cost and reprogram ability [7]. Without reasonably designed system software architectures and hardware structures, it is impossible to handle these tasks efficiently, safely and reliably, with the possibility of online reconfiguration and flexibly embedding applications.

Designing, monitoring and controlling such systems is becoming more and more challenging as a consequence of the steady growth of their size, complexity, level of uncertainty, unpredictable behavior, and interactions.

Moreover, SCADA systems have been applied in flexible manufacturing cells for educational purposes in different automation engineering fields [8]. Design and implementation of a low cost compact modular production system controlled by a SCADA system was developed for educational purposes [9]. In this way, students have access to instruments in a lab via the Internet, all by improving their skills related to SCADA systems, used in industry [10].

We present in this part some applications of SCADA systems design that have been presented in various researches.

Researchers, Ponsa P. \& al. [11], have described how Human-Machine-Interfaces have received appropriate attention in order to improve the design of SCADA applications in industrial domain. It is because of a major concern about aspects related to maintenance, safety, achieve operator awareness, etc has been gained. Even there are in the market software solutions that allow for the design of efficient and complex interaction systems, it is not widespread the use of a rational design of the overall interface system. The researchers have provided an example of such development also by showing how to include the automation level operational modes into the ergonomic interfacing system.

Researchers, Gezer D. \& al. [12], have presented a methodology and a case study through which system architecture and dynamic models of related system components are identified in order to design and simulate the SCADA system of a new hydro turbine test laboratory. System architecture model is prepared in System Modeling Language, a system modeling language based on Unified Modeling Language, while the dynamic model of the laboratory is formed in Matlab/Simulink. Some simulations are performed in order to verify the preliminary system design studies and system requirements.
Researchers, Dong W. \& al. [13], have presented a remote monitoring system that has been designed by using SCADA. Specifically, the monitor signals of boiler dry spot's worker are been composed first, and a real time database is realized on the basis of the collected information. In summary, these approaches could extend the system function and optimize the system structure. More importantly, it obtains the real-time data of web monitor system, which can draw the real-time curve of the dynamic systems and the curve of history, etc. Furthermore, it could achieve real-time brushless description.

Researchers, Morosan A. \& al. [14], have proposed an architecture for the SCADA system, used for making wellorganized production in manufacturing system, in order to can combine all the elements of a flexible manufacturing line. The main characteristic of this SCADA system is to supervise and to control the manufacturing process from a flexible manufacturing line.

Researchers, Chun-Lien S. \& al. [15], have proposed an approach to take the system performance into account in the reliability analysis of the SCADA system. This approach is based on a model for evaluating data transmission time, which allows us to find the operation time needed to complete SCADA functions. With information on performance, the reliability evaluation technique using fault tree analysis is described and applied for analysis of SCADA system component connectivity to assess the availability of SCADA controls. A sensitivity analysis is also described and used to illustrate the effects of input data uncertainty on the system reliability.

Researchers, Guobing H. \& al. [16], have studied an embedded SCADA system in order to manage and control industry objects directly at the production line terminal. A solution is recommended for an embedded SCADA system based on the development of embedded computer and network communication. The design and implementation methods are introduced for technical structure, hardware and software of this system. The first 10 units have been brought into use at power plants and power monitor centers since 2010. The result of site acceptance indicates that measuring precision and reliability follow correlative standards.

In the following part, we present the two structured analysis methods.

\section{PRESENTATION OF STRUCtURED ANALYSIS METHODS}

This section presents two structured analysis methods used in this research. These methods are: Structured Analysis Design Technique (SADT) and Structured Analysis Real Time (SA-RT). SADT, which was designed by Ross in the 1970s [17], was originally intended for software engineering but quickly other areas of application were found, such as training, operations, manufacturing, finance, etc. Although SADT (Figure 1) does not require any specific supporting tools, a number of computer programs implementing SADT methodology have been developed. Among of them is Design: IDEF, which implements IDEF0 method [18]. Furthermore, SADT/IDEF0 is a confirmed way to model any kind of domain [19]. 


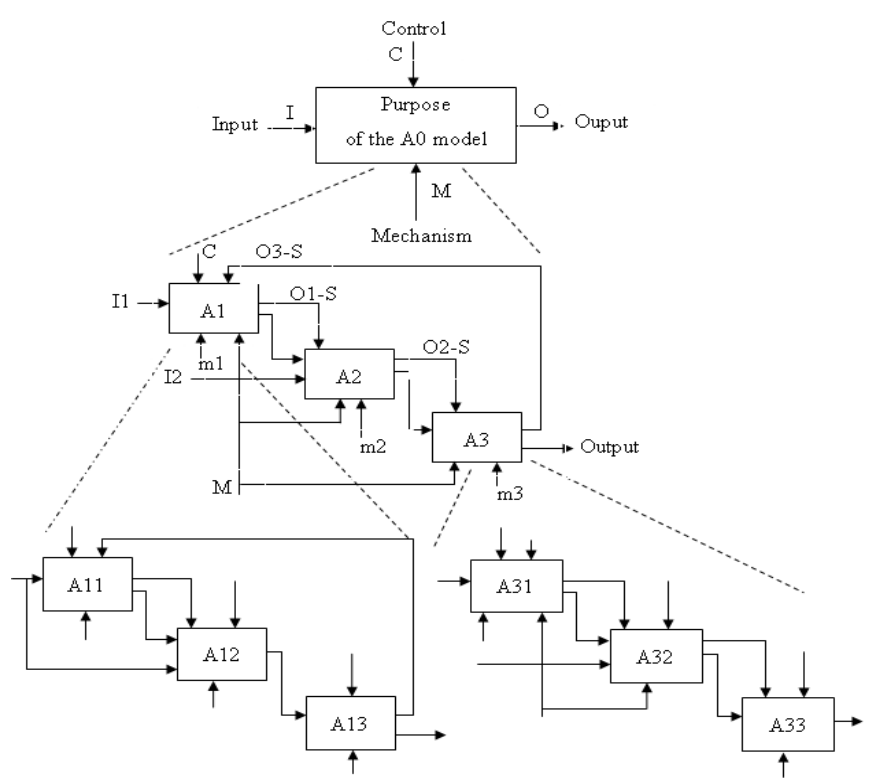

Fig. 1. Top-down, modular and hierarchical decomposition of SADT

Researcher, DeMarco, one of the pioneers of structured analysis (SA), described the need for such a method more than 30 years ago. He suggested that the products of analysis must be maintainable; problems of size must be dealt with using the effective method of partitioning; graphics must be used whenever possible [20]. Then, he proceeded further to establish requirements for the SA method as follows: the method should help us partition our requirements and document that partitioning before specification; it should give us means of keeping track of and evaluating interfaces; it should facilitate the development of new tools to describe logic and policy better than narrative text.

The notation used for creating SA diagrams consists of:

Data Flow Diagrams (DFDs): these diagrams model the processing of information in terms of data flows; data processing nodes are defined to represent the data processing functions from the requirements of the system.

Control Flow Diagrams (CFDs): these diagrams model the processing of information in terms of control flows; control nodes are defined to represent the control functions from the requirements of the system under analysis.

Process specifications: these specifications are used to describe the details of the data processing nodes defined in a DFD. These specifications consist of scripts of pseudo-code or just plain text which explains how the output flows of a particular processing node is generated from its input flows.

Control specifications: these specifications are used to describe the details of controls nodes in a CFD. These specifications define the behavioral model of the controllers and specify how the output control flows are obtained from the input control flows. They also specify when the data processing nodes are activated or deactivated.

Data Dictionary: it defines all the information flows and the data and control stores in the system. It contains text that defines each information item and its value range.
Entity-Relationship Diagrams (ERDs): these diagrams provide an information model for the data items and control signals and the relationships among these data items.

Figure 2 shows three dimensional views of the SA model. The top layer, which is the highest level in the SA-RT model, consists of what is commonly termed as the context diagram (CD). It describes the boundary of the software under analysis as well as the external interfaces and the external entities.

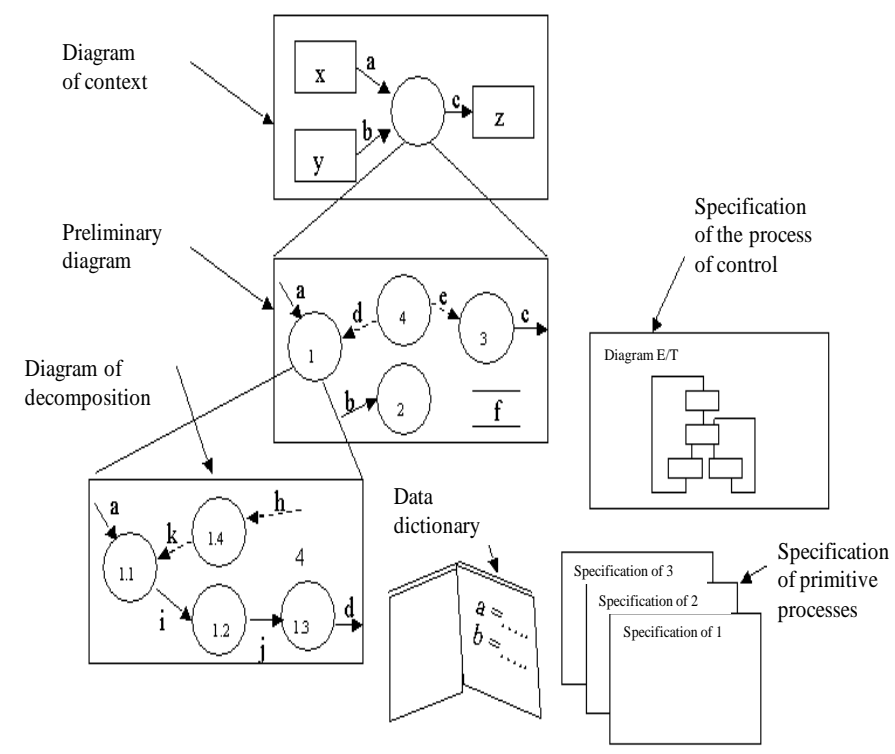

Fig. 2. Components of SA-RT and their relationship with each other

The following level of the hierarchy is a DFD which represents the major functions outlined in the functional requirements. These functions represent a top level decomposition of the software under developed.

Consequently the whole DFD0 is viewed as the child of the process representing the system in the top level. Each process in DFD (numbered 1, 2, 3, etc.) will have either its own child DFD or a P-spec sheet describing it in more detail. Also C-spec sheets are used to specify the controllers shown in each DFD.

We present in this part some applications of the SADT and SA-RT methods that have been presented in various researches:

Researchers, Benard V. \& al. [21], have described the SafeSADT method that enables the explicit formalization of functional interactions, the recognition of the characteristic values affecting the dependability of complex systems, the quantification of the reliability, availability, maintainability, and safety parameters of the system's operational architecture, and its validation in terms of the dependability objectives, as well as constraints set down in the functional requirement specifications.

Researchers, Lauras, M. \& al. [22], have presented an approach based on GRAI, SADT/IDEFO that enables the integration of the best practices defined by these methods. In addition the indicators next the results and determinants, three types of indicators are introduced to analyse the performance: the facility viewpoint measures, the appropriateness of the resources available and the determinants of the activity. 
Researcher, Marca D.A. [23], has explained how SADT/IDEF0 domain modeling can bring precise and complete context, to today's commonplace disciplines of the UML (Unified Modeling Language), Agile System Development, and Usability Engineering methods. In fact, the power and the rigor of SADT/IDEF0 come from: (1) a synthesis of graphics, natural language, hierarchical decomposition, and relative context coding, (2) distinguishing controls from transformations, (3) function activation rules, and (4) heuristics for managing model complexity.

Researchers, Jimenez F. \& al. [24], have developed models and tools for system design and synthesis of MEMS-micro based on SDL (specification description language), SA-RT and PNs. In fact, a main problem concerns the design of these varied circuits because it associates disciplines such as electronics, mechanics, chemistry, etc.

\section{GENERAL MODEL OF ANALYSIS AND SUPERVISION}

We present in this part, the development of a general model for the FA and the supervision of a production system in a SCADA environment.

The objective of the work is therefore the establishment of a general methodological gait of functional modeling and supervision of the production systems permitting to reach the aimed objectives [35].

The proposed model articulates around three essential phases: functional analysis of production systems; analysis of control-command applications; supervision of the controlcommand applications in a SCADA environment.

\section{Phase 1: Functional analysis of the production systems}

The first phase consists in proceeding to a functional modeling of the production systems while using the SADT method. The structure of this method permits to master the complexity of the process thanks to its downward and modular analysis.

The different stages of such a FA by the SADT method of the production systems are:

- To prepare the SADT model: the creation of a SADT model starts with the definition of two concepts that is the goal that stationary objectives of the model and the point of view that establish for what auditorium model it is created. These two determined concepts permit to guide the decomposition of every box;

- To create the diagram A-0 representing the general activity to analyze in an actigram;

- To create the diagram of activity A0: one writes down inside boxes drawn on a new form (3 to 6 boxes) the name of activities and one draws arrows of interfacing between the different boxes;

- From the diagram A0, to select the least complex box and that will give more information to create the diagram by decomposition have $(1<\mathrm{i}<6)$ with corresponding $\mathrm{i}$ to the number of the box in $\mathrm{A} 0$;
- To redo the same principle of decomposition for the other diagrams of activity;

- To represent all diagrams of the model on the SADT forms.

This first phase of the model enables us the decomposition of the production system under a hierarchized manner to bring back it to the elementary situations and to elaborate a static model describing the activities of the process.

\section{Phase 2: Analysis of the control-command applications}

Once functions and activities of every function have been identified, the following stage consists in analyzing controlcommand applications while using the SA-RT method.

The different stages of such a FA by the SA-RT method of control-command applications are:

- To establish the CD while representing the different terminations as well as the incoming and retiring data streams;

- To establish the preliminary diagram (DFD0) that represents the necessary functional process list to the application with the stream of data correspondents.

- To establish the diagram of decomposition partner streams to one of the functional processes identified;

- To establish the DFC representing the controls aspect the SA-RT method;

- To establish the State/Transition diagram representing the behavior aspect or real time of the application;

- Functional process specification (dictionary of data) while leaning on a procedural specification.

This second phase of the model enables us the decomposition of the studied application under a hierarchized manner to bring back it to the elementary situations and to drive to the development of a dynamic model describing the various processes of the application as well as flows of data and control.

Phase 3: Supervision of the control-command application in a SCADA environnement

After a various process analysis and flows of data and control of the application, different static and dynamic model are elaborated. The last stage of the model of the analysis and the supervision of the production system consists in the establishment of tools and methods of supervision, control and date acquisition using a SCADA system as well as the development of Man - Machine interfacings. In fact, the supervision is the whole of tools and methods that permit to drive some industrial facilities so much in normal working that in presence of failings. It is the tool of reference of the conduct operator but can interact also directly with the controlcommand application.

The supervision has for objective to supervise and to control the working of an installation so that it remains in the normal working whatever are the outside disruptions. It permits 
to detect in real time mistakes from alarms and to identify their reasons.

In the following part, we present the results of FA and supervision on an example of a SCADA system of a thermal power plant (TPP) as well as the development of different phases of the general model proposed.

\section{RESUlTS OF FUNCTIONAL ANALYSIS AND SUPERVISION}

In this section, we present on the one hand an example of a SCADA system in a TPP in Tunisia and on the other hand a control-command application of the water steam cycle of the TPP. By means of a significant example, the objective of this work is to validate the general model of analysis and supervision presented in the previous section [31], [32].

\section{A. Presentation of the SCADA system and a case study of a water steam cycle}

In Tunisia, the TPP of Rades (near to Tunis) is composed of thermal power stations that are one of the most important stations of the electric energy production in Tunisia (37\% of the national production) [33], [34]. In fact, the SCADA system is used by the power station operators in order to supervise the good working of two production slices of the TPP. This centralized supervision permits to operators, since the control room to control facilities in their domain of exploitation and to treat, in real time, the different types of incidents (Figure 3).

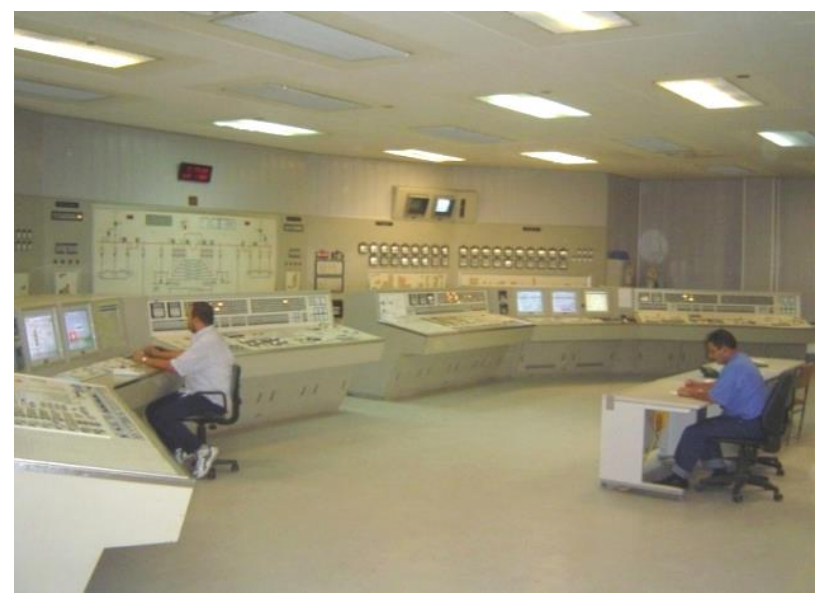

Fig. 3. Control room of the TPP

The electric energy production in the TPP of Rades is based on a set of energies transformations using water as energy support. This water must have a high quality in order to guarantee the security of the installation and to improve the production groups' performances. It is therefore necessary to apply a rigorous treatment of the raw water and a stern control of its quality. TPP.

Figure 4 shows the architecture of the SCADA system of a

The pretreatment is constituted of two filtration chains each including a sand filter and an active coal filter. Thereafter, the water passes by the filtration chain then introduced in the inverse osmosis station and thereafter in the demineralization station (Figure 5).

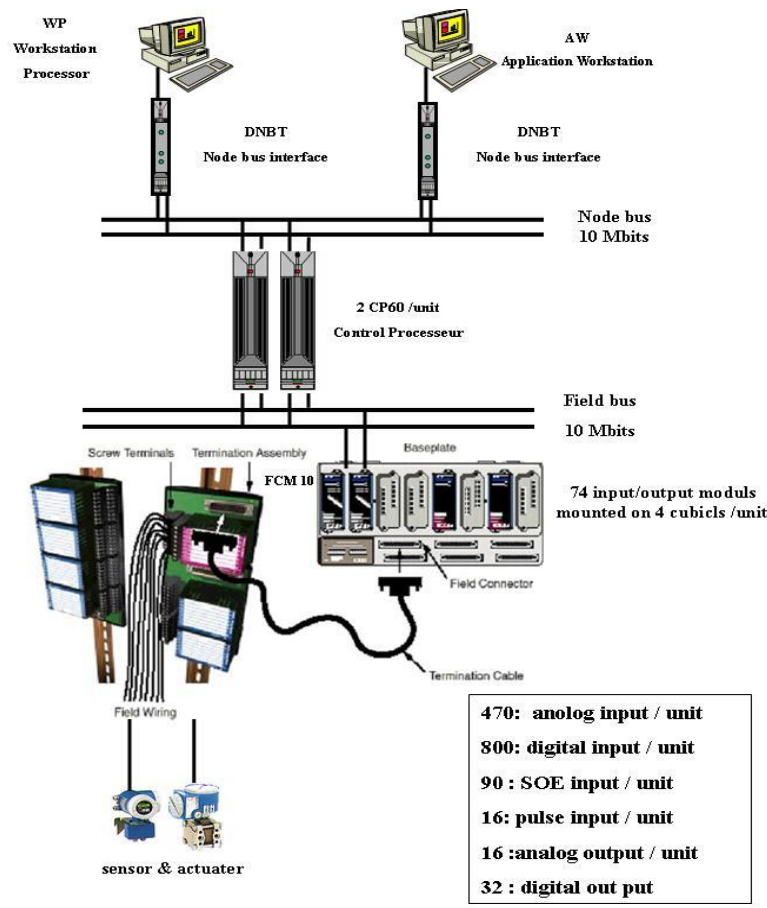

Fig. 4. SCADA system of the TPP

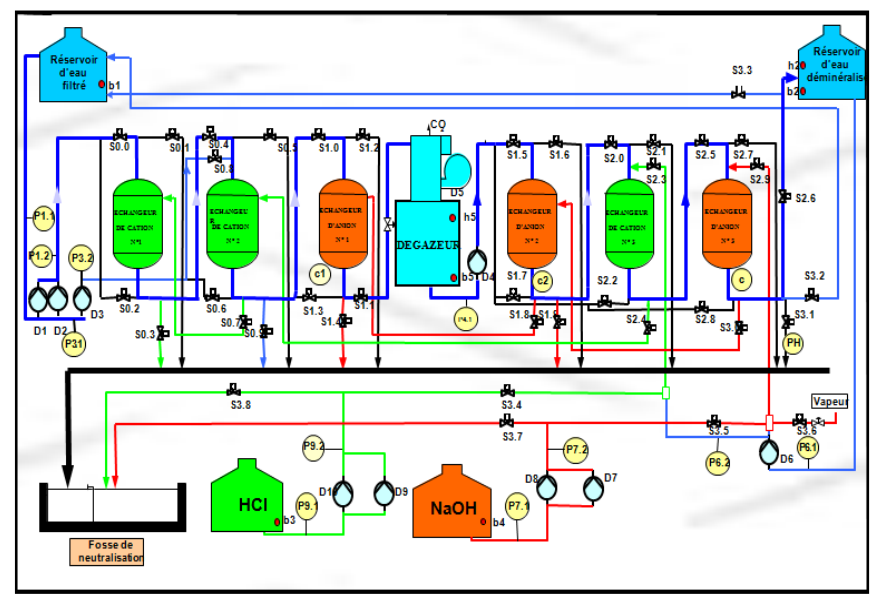

Fig. 5. Demineralization station of the TPP

\section{B. Results of analysis}

According to the first phase of the methodology established, a FA using the SADT method has been done (Figure 6). This analysis is a very interesting stage because it permits to describe the electric energy process of the TPP.

The second phase of the proposed methodology consists on the use of the SA-RT method for the analysis of a controlcommand application of the TPP.

Figure 7 shows the CFD of the water-steam station of the TPP. 


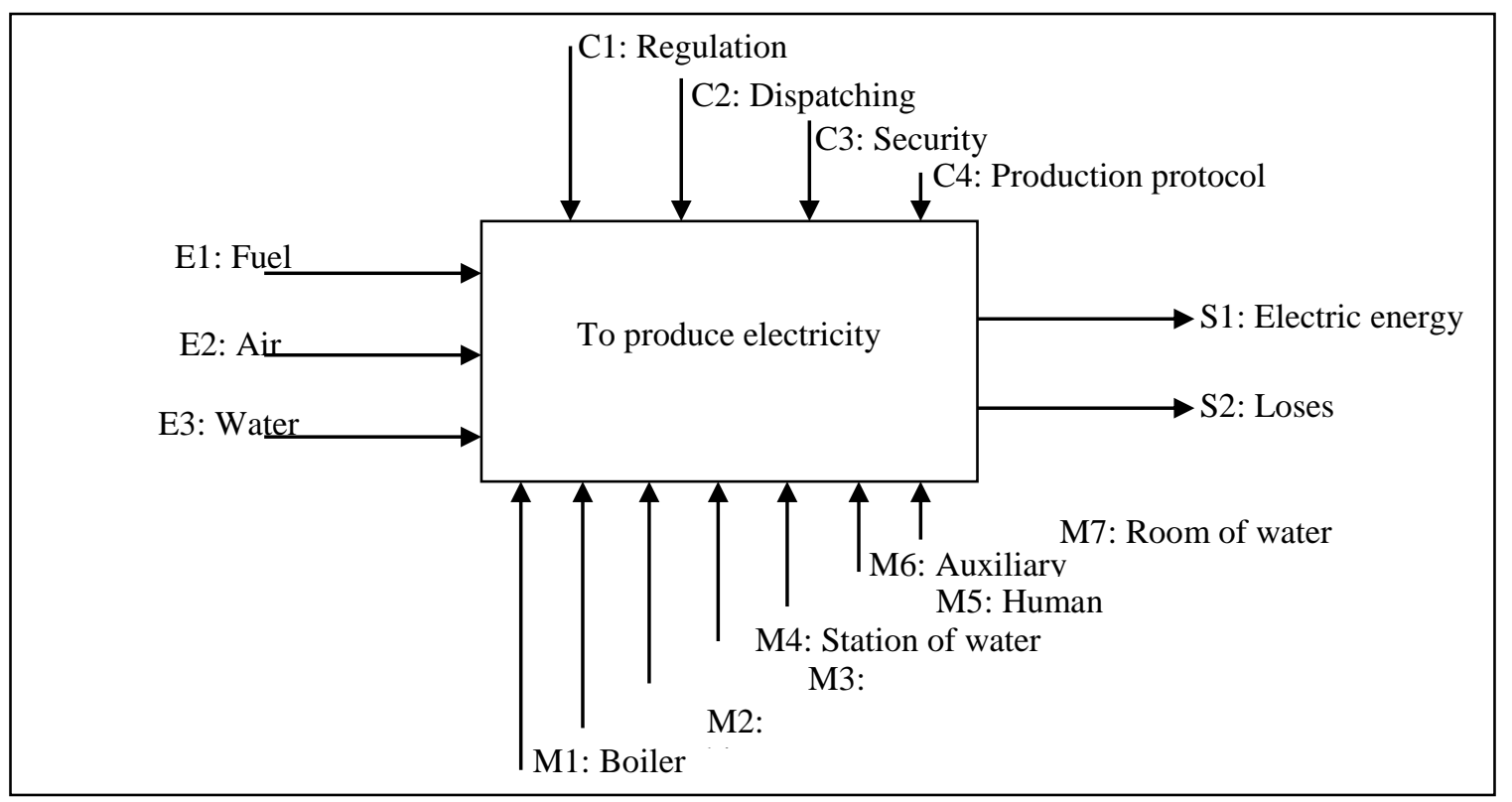

Fig. 6. Node A-0 of the SADT model of a TPP

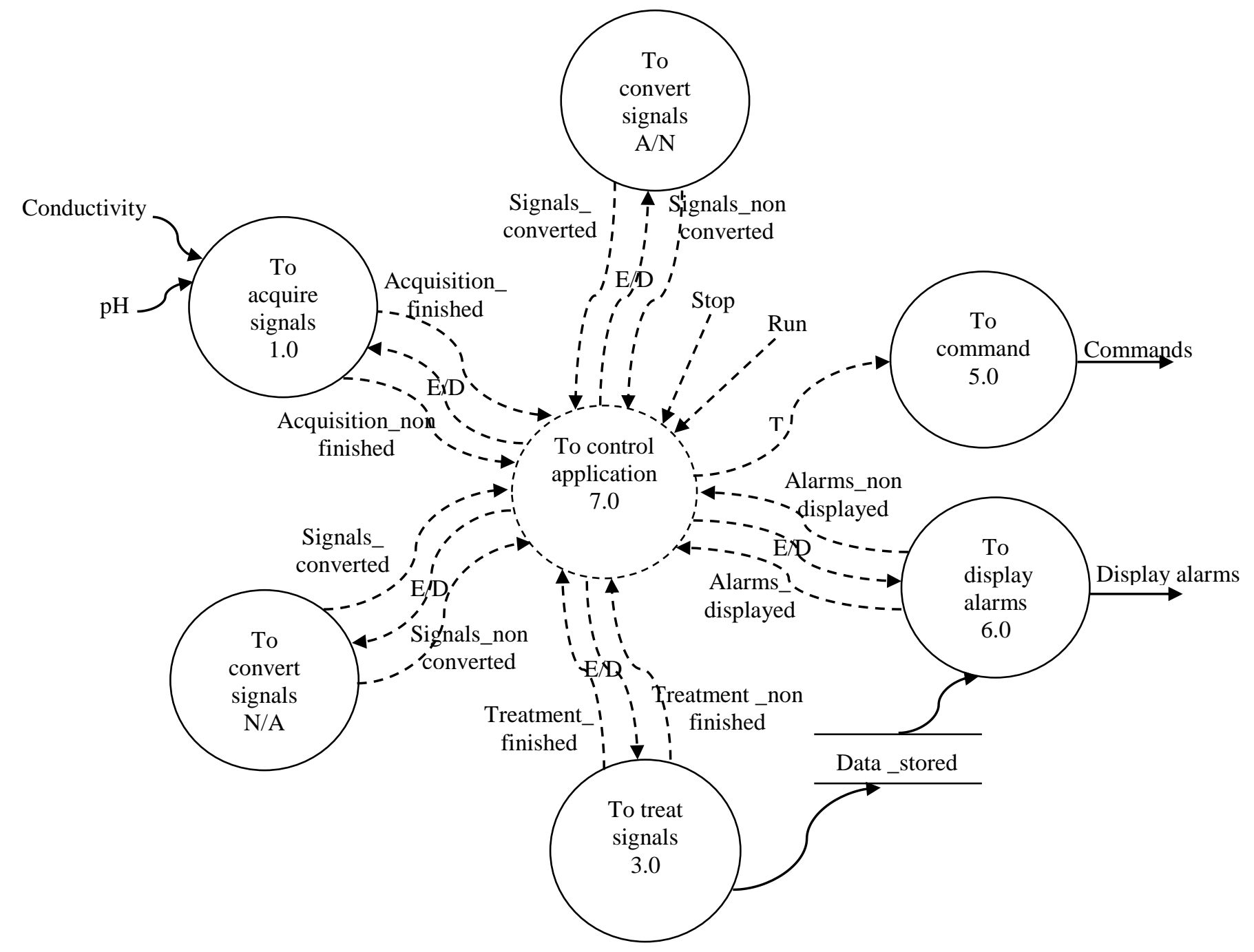

Fig. 7. Control Flow Diagram of the SCADA system of the water-steam station 
The CD of the SA-RT model is constituted of one functional process «To pilot the control-command application $0 »$ and the terminators. It defines perfectly the interface between the designer and the client, that is, to provide or generate data in order to display these data on the tabular of the control-command application.

The DFD of the SA-RT model constitutes the first decomposition of the process presented in the $\mathrm{CD}$. Then, we can identify the initial functional processes of the control-

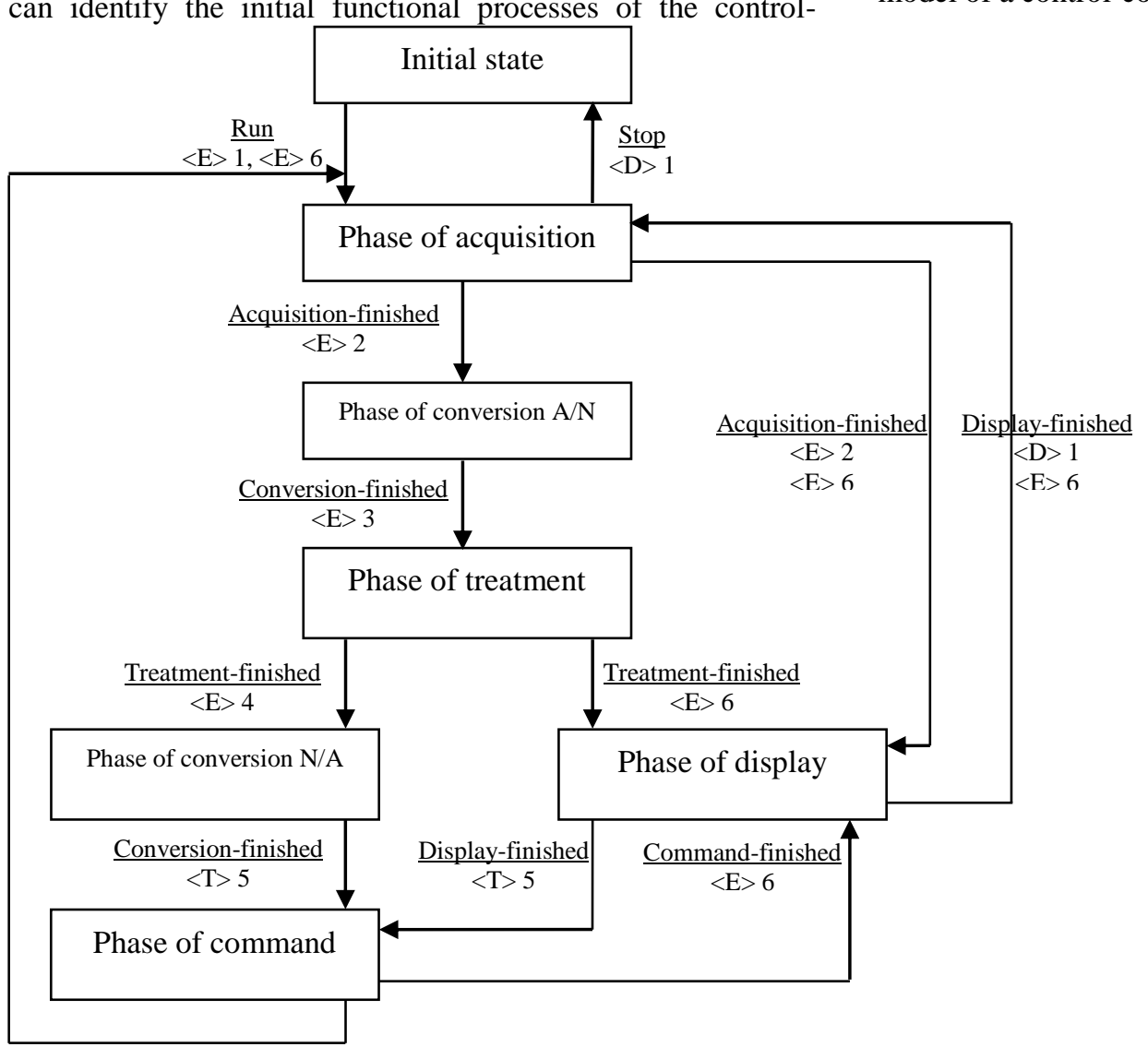

command application: acquirement process; treatment process; Human-Machine-Interfaces process.

The CFD of the SA-RT model includes the control aspect to the DFD elaborated. In fact, the implementation of the monitoring process at the level of preliminary diagram can express the sequence execution of the functional processes.

Figure 8 shows the state-transition diagram of the SA-RT model of a control-command application.

Fig. 8. State/Transition diagram of a SCADA system of the water-steam station

The potential uses for the SA-RT model are the design of the monitoring display and the diagnosis display. For the design of a monitoring display, the preliminary DFD of the SA-RT model presents an overall view of the controlcommand application. Indeed, information relative to each process represented through this level should appear in the monitoring display.

For the design of hierarchical diagnosis display, each DFD of the SA-RT model constitutes a vision at a given abstraction level. So, each of these DFDs gives a less or more detailed vision. In function of the objectives defined by the designer for each display, a particular DFD can supply the required information.

Finally, this application of the SA-RT method on the SCADA system of a TPP shows briefly the interests of the FA the design of supervisory systems.

\section{Results of supervision}

The last phase of the methodology proposed consists on the supervision of the control-command application. For the example of the water-steam cycle, we study the interfacing of the different signals: $\mathrm{pH}$ and conductivity of the ball furnace of the TPP. The application is declined in six stages: 1) choosing the site of the signal (FBM module); 2) programming both AIN and CIN blocks for the supervision of the signals $\mathrm{pH}$ (4 to 20 $\mathrm{mA}$ ) and conductivity (alarm); 3) testing both AIN and CIN blocks by injection of current and by short circuit; 4) passing the cable between the sampling room and the SCADA room; 5) connecting the signals in the two modules 10FBM215 and $10 \mathrm{FBM} 325$; 6) conceiving a new tabular for the general vision of the sampling room.

Figure 9 shows the display of the sampling room containing the chemical analysis parameters of the water-steam cycle. 


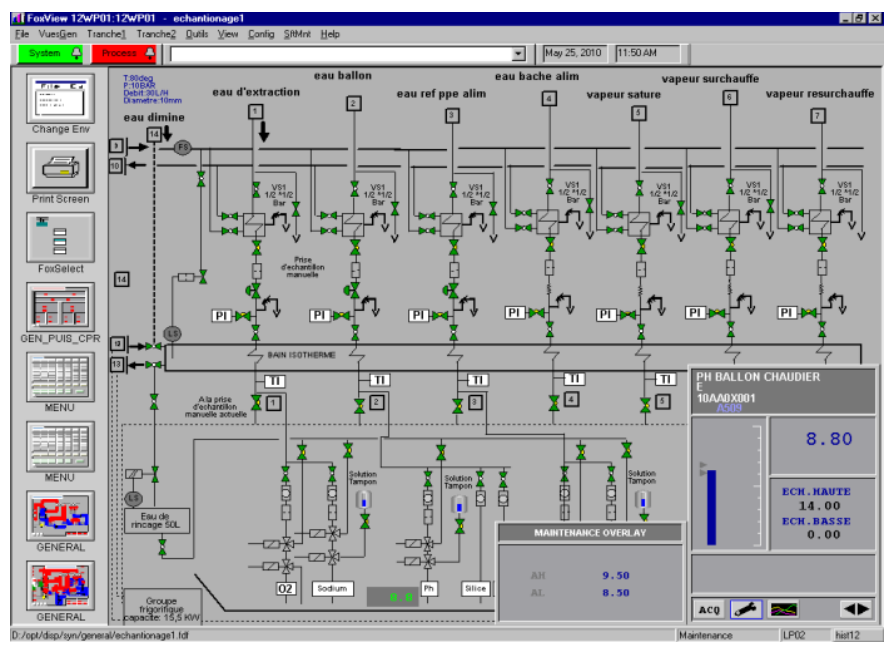

Fig. 9. Display of the chemical analysis parameters

In the first step is used the FoxView software which represents the interfacing operator to visualize the synoptic with a menu bar to activate the main functions of the SCADA system. Then, in the second step is used the ICC (Integrated Control Configurator) to create and to configure programs residing in the $\mathrm{CP}$ (Control Process).

To access ICC, we need from the menu bar Config choose term Control_Cfg-CIO_STN_Cfg and Config_station_name. It is used to configure the various blocks AIN, CIN, CALC, etc. Indeed, during the programming of a new signal it must be identified: the signal label; the compound and the address signal. For simulation is used FoxSelect software that permitting to reach the various elements of the hierarchy of data base of the CP.

The last step of this application is to improve the synoptic the $\mathrm{pH}$ meter and the conductivity meter and the design of a new synoptic of the sampling room containing these two data from the Fox Draw software. It provides tools to design displays for monitoring, alarming and process control.

To access FoxDraw we can choose function ConfigFoxDraw from the menu bar. Included with FoxDraw is a large library of graphical components ready to be integrated and configured in displays. Thus, we proceeded as follows: creating of $\mathrm{pH}$-meter blocks; operating test of the $\mathrm{pH}$-meter; display configuration of different alarms; configuring of the overleay $\mathrm{pH}$ and testing the overleay $\mathrm{pH}$ - meter.

\section{CONCLUSION}

In order to understand our world we need to think that almost everything can be regarded as a system, where signals must be monitored, controlled and ultimately supervised. In fact, the supervision of the production systems should by nature try to guarantee the observability, the controllability and, most important, the system stability.

In this paper, we presented a methodology of analysis and supervision of the production systems. We have suggested three main directions to achieve that methodology: (1) functional analysis of the production systems; (2) analysis of the control-command applications; and (3) supervision of the control-command applications in a SCADA environment. Two interesting functional analysis methods SADT and SA-RT are used on the one hand and a SCADA system for monitoring, control and fault tolerance, on the other hand. In fact, allowing the running of the production equipment to be understood, these techniques permit designers to decide the good information to display through the supervisory interfaces devoted to each type of supervisory task (monitoring, diagnosis, action...). In addition, functional analysis techniques might be a good assist to design support systems such as alarm filtering systems.

Staring from this case study of the analysis and supervision of the production systems discussed in this paper, work is in progress to develop a functional analysis and real time for different control-command applications in different production systems.

\section{REFERENCES}

[1] M. Lambert, B. Riera and G. Martel, Application of functional analysis techniques to supervisory systems, Reliability Engineering and System Safety 64, 1999, pp. 209-224.

[2] D.J. Hatley and I.A. Pirbhai, Stratégies de spécification des systèmes temps réel (SA-RT), Masson, Paris, France, 1991.

[3] F. Cottet, Systèmes temps réel de contrôle - commande, Dunod, Paris, 2005.

[4] M.N. Lakhoua, SCADA applications in thermal power plants, International Journal of the Physical Sciences, Academic Journals, vol.5, $\mathrm{N}^{\circ}$, 2010, pp. 1175-1182.

[5] M.N. Lakhoua, Surveillance of pumps vibrations using a SCADA, Control Engineering and Applied Informatics, Romanian Society of Control Engineering and Technical Informatics, vol.12, N ${ }^{\circ} 1,2010$.

[6] Y.L. Kaszubowski, R.S. Rosso, A. Leal, E. Harbs and M.S. Hounsell, Finite Automata as an Information Model for Manufacturing Execution System and Supervisory Control Integration, Proceedings of the 14th IFAC Symposium on Information Control Problems in Manufacturing, Bucharest, Romania, May 23-25, 2012.

[7] E.I. Gergely, Dependability Analysis of PLC I/O Systems Used in Critical Industrial Applications, Sudies in Computational Intelligence, 417, Springer, 2013, pp. 201-217.

[8] S. Reynard, O. Gomis, F. Bellmunt, A. Sudrià, O. Boix, and I. Benítez, Flexible manufacturing cell SCADA system for educational purposes, Comput Appl Eng Educ 16, 2008, pp. 21-30.

[9] R. Yenitepe, Design and implementation of a SCADA controlled MTMPS as a mechatronics training unit. Comput Appl Eng Educ 20, 2012, pp. 247-54.

[10] Z. Aydogmus and O. Aydogmus, A web based remote access laboratory using SCADA, IEEE Trans Educ, 52, 2009, pp. 126-132.

[11] P. Ponsa, R. Vilanova , A. Pérez, and B. Andonovski , SCADA design in automation systems, 3rd Conference on Human System Interactions (HSI), 2010, pp. $695-700$.

[12] D. Gezer, H.O. Unver , Y. Tascioglu , K. Celebioglu and S. Aradag, Design and simulation of a SCADA system using SysML and Simulink, International Conference on Renewable Energy Research and Applications (ICRERA), 2013, pp. 1058-1062.

[13] W. Dong and S. Xian-li, The boiler design of remote monitoring system based on the SCADA, Chinese Automation Congress (CAC), 2013, pp. 864- 869.

[14] A. Morosan and F. Sisak, A SCADA system designed for making more efficient production in flexible manufacturing system, IEEE 13th International Symposium on Computational Intelligence and Informatics (CINTI), 2012, pp. 409- 413.

[15] S. Chun-Lien and C. Ya-Chin, A SCADA system reliability evaluation considering performance requirement, International Conference on Power System Technology, 2004, vol.1, pp. 574-579. 
[16] H. Guobing, M. Guoqiang, Z. Jianmin and D. Jianling, Design for an embedded SCADA system, International Conference on Electrical and Control Engineering (ICECE), 2011, pp. 5819-5821.

[17] D.T. Ross, Structured Analysis (SA): A language for communicating ideas, IEEE Transaction on Software Engineering, 3(1), 1977, pp. 16-34.

[18] IEEE 1320.1-1998. IEEE Standard for Functional Modeling LanguageSyntax and Semantics for IDEF0, IEEE, 1998.

[19] M.N. Lakhoua, Systemic analysis of an industrial system: case study of a grain silo, Arabian Journal for Science and Engineering, ISSN: 13198025, vol.38, 2013, pp. 1243-1254.

[20] T. DeMarco, Structured Analysis and System Specification, PrenticeHall Inc., New Jersey, 1979.

[21] V. Benard, L. Cauffriez and D. Renaux, The Safe-SADT method for aiding designers to choose and improve dependable architectures for complex automated systems, Reliability Engineering \& System Safety, 93(2), 2008, pp. 179-196.

[22] M. Lauras, J. Lamothe, and H. Pingaud, Une méthode orientée processus pour le pilotage par la performance des systèmes industriels, Journal Européen des Systèmes Automatisés, 41(1), 2007, pp.71-100.

[23] D.A. Marca, SADT/IDEF0 for Augmenting UML, Agile and Usability Engineering Methods, Marca, David., 2012, Software and Data Technologies, pp. 38-55.

[24] F. Jimenez, M. Courvoisier, A. Garcia, G. Munoz , N. Harchani, M. AlMohamed and D. Esteve, Tools and models for systems design and synthesis of MEMS based on asynchronous circuits, IEEE International Conference on Industrial Technology, vol.1, 2000, pp. 64- 69.

[25] I. Kuuluvainen and V. Ylitolva, The Bubble Operating System: a control extension to data flow diagram, IEEE Transactions on Consumer Electronics, 1991, Vol.37, Issue: 3, pp. 642- 650.
[26] A. Flo, M. Kjaernes and A. Skomedal, A bridge from structured analysis (SA/RT) to specification and description language (SDL), Eighth International Conference on Software Engineering for Telecommunication Systems and Services, 1992, pp. 93-97.

[27] N.Sahraoui, Applying specification methods to complex systems, IEEE International Conference on Systems, Man, and Cybernetics, vol.5, 1997, pp. 4488- 4491.

[28] L. Urbain and B. Tondu, Robot controller specification using SART approach, Proceedings of the Third IEEE Conference on Control Applications, 1994, pp. 303-308.

[29] S. Lihua and J.A. Keane, Algorithmic aspects of hierarchical verification for SA/RT models, IEEE International Conference on Computational Cybernetics and Simulation, vol.3., 1997, pp. 2252- 2257.

[30] R.B. France and J. Bruel, Using Integrated Formal and Informal Modeling Techniques to Analyze Software Requirements: A PetriNet/SART Case Study, 1996.

[31] M. Ben Hammouda, M.N. Lakhoua and L. El Amraoui, Dependability evaluation and supervision in thermal power plants, International Journal of Electrical and Computer Engineering, vol. 5, $\mathrm{N}^{\circ} 5$ : October 2015.

[32] M.N. Lakhoua and H.Laadhari, Supervision of the natural gas station using a SCADA System, Journal of Electrical and Electronics Engineering, vol.6, $\mathrm{n}^{\circ} 1$, May 2013.

[33] M. Ben Hamouda and M.N. Lakhoua, Methodology of Operating Safety and Supervision of a Production System, CISTEM, IEEE, 3-6 Nov. 2014, Tunisia.

[34] R. Glaa and M.N. Lakhoua, Methodology of Analysis and Design of a SCADA System, CISTEM, IEEE, 3-6 Nov. 2014, Tunisia. 